\title{
Black raspberries in cancer clinical trials: Past, present and future
}

\author{
Laura A. Kresty ${ }^{\mathrm{a}, *}$, Susan R. Mallery ${ }^{\mathrm{b}}$ and Gary D. Stoner ${ }^{\mathrm{a}}$ \\ ${ }^{a}$ Department of Medicine, Division of Hematology and Oncology, Medical College of Wisconsin, \\ Milwaukee, WI, USA \\ ${ }^{\mathrm{b}}$ Division of Oral and Maxillofacial Pathology and Radiology, College of Dentistry, \\ The Ohio State University, Columbus, OH, USA
}

Received 18 December 2015; accepted 27 March 2015

\begin{abstract}
.
BACKGROUND: Black raspberries (BRB) inhibit a broad range of cancers in preclinical models, including in vivo models of oral, esophageal, colon, breast and skin cancer. Promising preclinical results have led to clinical evaluations in cancer patients or patients at increased risk for cancer development.

OBJECTIVE: To summarize clinical investigations targeting cancer or precancerous lesions with BRB and discuss future directions.

METHODS: A thorough literature search was conducted through December 1, 2015 to identify all published studies evaluating BRB in cancer focused clinical trials.

RESULTS: Research investigating BRB in clinical settings report positive effects on preneoplastic lesions or cancers of the oral cavity, esophagus and colon. BRB treatment resulted in: histologic regression of oral intraepithelial neoplasia associated with improved histologic grade and significantly reduced loss of heterozygosity at tumor suppressor gene loci, modulated genes linked to RNA processing and growth factor recycling; in the colon, BRB inhibited FAP-associated polyp progression, demethylated tumor suppressor genes and improved plasma cytokine profiles; in Barrett's patients, BRB consumption increased tissue levels of GST-pi and decreased 8-isoprostane, a marker of lipid peroxidation/oxidative stress.

CONCLUSIONS: The precise dose, duration and optimum mode of BRB delivery for cancer inhibition remains to be fully elucidated. Common themes across studies support that BRB are anti-proliferative, anti- inflammatory, reduce oxidative stress and restore tumor suppressive activity. Future directions are included in the conclusions section.
\end{abstract}

Keywords: Cancer prevention, black raspberry, oral cavity, esophagus, colon, human clinical trial

\section{Introduction}

Over the last decade a numerous lines of evidence have converged to support clinical investigations utilizing black raspberries (BRB) as inhibitors of cancer or premalignancy in high risk human cohorts. First, mounting epidemiological evidence shows increased consumption of plant based diets is associated with decreased cancer risk, particularly cancers of the aerodigestive tract [1-4]. Second, strong preclinical results in animal models report that $\mathrm{BRB}$ inhibit cancers of the oral cavity, esophagus, colon, breast and skin through targeting processes

\footnotetext{
${ }^{*}$ Corresponding author: Laura A. Kresty, Medical College of Wisconsin, Division of Hematology \& Oncology, Department of Medicine, TBRC \#3910, 8701 Watertown Plank Road, Milwaukee, WI 53226, USA. Tel.: +1 414955 2673; E-mail: Lkresty@mcw.edu. 
of proliferation, inflammation, angiogenesis and apoptosis [5-14]. Third, from a composition stand point black raspberries are rich in vitamins, minerals, fiber, anthocyanins, total phenolics and other bioactive components with cancer inhibitory capacity as previously reviewed [15]. Lastly, initial research reported that maximum concentrations of the major anthocyanins and ellagic acid occurred at 1 to 2 hours in the plasma and between 30 minutes and 4 hours in the urine following BRB consumption with less than $1 \%$ overall uptake of the parent or precursor anthocyanins raising concerns regarding bioavailability; however, more recent research shows bioavailability of anthocyanins is comparable to other flavonoid subclasses with significant uptake of diverse metabolites with half-lives ranging from 2 to 96 hours [16]. In turn, the metabolites reportedly modify cellular adhesion and inflammatory signaling cascades, both important cancer associated processes [16].

\section{Materials and methods}

A thorough bibliographic search was conducted utilizing PubMed through December 1, 2015 to identify all published clinical interventions utilizing black raspberries to target cancers or premalignant lesions. Keywords searches included black raspberry, black raspberries, cancer, premalignant, premalignancy and clinical trials in combination to ensure all completed published investigations were identified. Clinical research with BRB has been completed targeting cancer or premalignancy of the oral cavity, esophagus and colon [17-25] and will be reviewed herein. Individual clinical trial details are reported or referenced in the section summarizing each research investigation.

\section{Results}

\subsection{Pilot study of BRB gel in patients with oral premalignancy}

An estimated $60 \%$ of cancers of the mouth, pharynx and larynx may be prevented by eliminating tobacco use, reducing alcohol intake and increasing consumption of fruits and non-starchy vegetables [4, 26, 27]. Among never smokers, high fruit consumption is strongly protective against head and neck cancers. Mallery and colleagues designed two studies which effectively build upon epidemiological findings and extend positive preclinical research into clinical trials in at risk patients with oral premalignancy [17, 18]. A 6 week trial was conducted utilizing a 10\% BRB gel to target oral premalignancy, ranging from hyperkeratosis with atypia to severe dysplasia [17]. Details of the gel preparation have been previously documented [17-19, 28, 29]. In brief, the gel contained $10 \%$ freeze dried black raspberry powder and was $\mathrm{pH} 3.5$ to stabilize the more biologically active flavylium cation of the anthocyanin molecules [17]. Patients applied the gel $4 \times$ daily delivering $0.5 \mathrm{~g}$ of BRB in each application. The study details and inclusion criteria which included patients 18 years of age or older with microscopically confirmed premalignant oral epithelial changes and no recent use of tobacco products have been previously published in two reports $[17,18]$. The first and second investigation evaluated 17 and 20 patients, respectively with oral premalignancy and 10 patients with histologically normal oral epithelium. The study approach included removing half of the premalignant lesional tissue pretreatment or in patients with normal epithelium, normal tissue was removed from the ventral lateral tongue. Patients were re-biopsied following the 6 week treatment, including the residual treated site and the initial biopsy area [17]. Endpoint measurements included histologic diagnoses and loss of heterozygosity at tumor suppressor gene loci (INK4a/ARF, p53 and FHIT), which are altered in oral premalignancy [30] and associated with progression to squamous cell carcinoma [31, 32]. BRB administered as a berry gel for 6 weeks was well tolerated, as none of the patients reported adverse events and normal tissue epithelium from control patients remained normal [17]. Importantly, BRB gel treatment resulted in histologic regression in a subset of patients and resulted in a statistically significant reduction of loss of heterozygosity (LOH) prevalence [17]. Specifically, following BRB treatment $41 \%$ of subjects showed a decrease in lesional grade, $23 \%$ an increase in histologic grade and the remaining $35 \%$ no change in histologic grade [17]. 
Next further research was conducted in the same cohort, with 3 additional cases and expanded outcomes to include growth factor, proinflammatory and angiogenesis inducing enzymes (VEGF, COX-2, iNOS), gene expression profiles and microvessel density (MVD). Results showed that topical BRB gel suppresses genes associated with RNA processing, growth factor recycling, and inhibition of apoptosis [18]. In addition, COX-2 levels were significantly reduced post-BRB treatment [18]. BRB treatment reduced MVD, as measured by CD34, in $63.6 \%$ of patients; whereas, MVD levels increased in the remaining patients [18]. These results support that $\mathrm{BRB}$ gel has positive effects on histological regression, reduces $\mathrm{LOH}$, inflammatory markers and MVD; however, there seems to be a subpopulation of patients that are more responsive compared to other patients [18].

\subsection{Placebo controlled study of BRB gel in patients with oral premalignancy}

Based on the promising results utilizing a BRB gel over a 6 week time period in patients with oral premalignancy, an expanded multi-centered placebo controlled trial of 3 months in duration was conducted to further assess efficacy [19]. Forty patients with microscopically confirmed oral premalignancy were enrolled, 22 patients were randomized to the $10 \% \mathrm{BRB}$ gel treatment arm and 18 patients to the placebo gel arm. Topical application of BRB gel ( $0.5 \mathrm{~g} 4 \times$ daily) on oral premalignant lesions resulted in significant reductions in lesion size, histologic grade and $\mathrm{LOH}$ events. In contrast, lesions treated with placebo gel significantly increased in size without improvement in histologic grade or reductions in LOH levels [19]. Specifically, 70.6\% of placebo treated lesions increased in size; whereas, $76.2 \%$ of BRB gel treated lesions reduced in size. Among BRB treated patients $41 \%$ experienced decreases in lesion grade following 3 months of treatment. The study further revealed higher pretreatment levels of BRB metabolic and keratinocyte differentiation enzymes in patient lesions that were BRB responsive, pointing to inherent differences in metabolic and differentiation capacity among patients [19]. As discussed by the author's interpatient variation in responsiveness pose a real challenge in the context of prevention trials [19].

Additional research is needed to improve our understanding of absorption, metabolism and bioactivity of BRB and their constituent polyphenols on a per patient basis. Kay and colleagues recently employed ${ }^{13} \mathrm{C}$-labeling of cyanidin-3-glucoside (C-3-Gluc), a major BRB anthocyanin, as a novel approach to investigate pharmacokinetics and identify new metabolites following ingestion of C-3-Gluc [16]. Surprisingly, this targeted labeling approach revealed that anthocyanins have a minimum bioavailability of $12.4 \%$ on the basis of total elimination of absorbed ${ }^{13} \mathrm{C}$ dose [16]. This level is considerably higher than previous reports [33] and supports that anthocyanins are as bioavailable as other flavonoid subclasses. This study also reported high variation in the recovery of the ${ }^{13} \mathrm{C}$ tracer between subjects supporting large interindividual differences which in turn may impact compound fate and patient responsiveness to interventions, as noted in the oral cavity studies discussed [17-19]. New insight regarding absorption, metabolism and excretion can be derived from ${ }^{13} \mathrm{C}$-labeling studies and should be considered for other polyphenols of interest.

Moreover, a highly accessible location like the oral cavity offers a ready model for investigating controlled direct delivery of agents and determination of interpatient response variability. It permits more precise delivery in terms of dose, ease of administration multiple times a day, likely requires less total product for efficacy and may benefit from local metabolism. Preclinical studies support that BRB have been most effective where there is some direct contact with the target area, with the only exception being a single in vivo study in a preclinical model for breast cancer [13]. Thus, novel labeling approaches may prove useful for improved targeting of inhibitory agents and increasing our understanding of differential patient responsiveness.

\subsection{BRB in barrett's esophagus patients}

Barrett's esophagus (BE) is the only known precursor lesion for esophageal adenocarcinoma (EAC), a rapidly rising cancer with poor survival rates [34]. Reflux of gastric and duodenal contents, known as gastroesophageal reflux disease (GERD), is the main risk factor for BE and EAC [35, 36]. GERD frequently manifests as heartburn 
Table 1

Clinical trials of BRB targeting premalignancy or cancer

\begin{tabular}{|c|c|c|c|c|c|}
\hline $\begin{array}{l}\text { Population, no. } \\
\text { patients }\end{array}$ & $\begin{array}{l}\text { Route \& mode of } \\
\text { delivery }\end{array}$ & Dose & $\begin{array}{l}\text { Duration of } \\
\text { Study }\end{array}$ & Endpoints & References \\
\hline $\begin{array}{l}\text { Oral dysplasia } \\
(n=27 \text { and } 30)\end{array}$ & $\begin{array}{l}\text { Direct local delivery } \\
\text { in berry gel } \\
\quad \text { formulation } \\
\text { ( } n=17 \text { and } 20 \mathrm{w} / \\
\text { Premalignancy \& } \\
n=10 \text { Controls) }\end{array}$ & $\begin{array}{l}0.5 \mathrm{~g} \mathrm{BRB} \text { or } \\
10 \% \mathrm{w} / \mathrm{w} \\
4 \times \text { daily }\end{array}$ & 6 weeks & $\begin{array}{l}\text { Lesion size } \\
\text { Histopathology } \\
\text { Loss of } \\
\quad \text { heterozygosity } \\
\text { COX-2, iNOS, CD34 } \\
\text { Gene expression }\end{array}$ & $(17,18)$ \\
\hline $\begin{array}{l}\text { Oral dysplasia } \\
(n=30)\end{array}$ & $\begin{array}{l}\text { Direct local delivery } \\
\text { in berry gel } \\
\text { formulation }(n=22) \\
\text { or placebo }(n=18)\end{array}$ & $\begin{array}{l}0.5 \mathrm{~g} \mathrm{BRB} \text { or } \\
10 \% \mathrm{w} / \mathrm{w} \\
4 \times \text { daily or } \\
\text { placebo }\end{array}$ & 3 months & $\begin{array}{l}\text { Lesion size } \\
\text { Histopathology } \\
\text { Loss of } \\
\quad \text { heterozygosity } \\
\text { COX-2, iNOS } \\
\text { Gene expression }\end{array}$ & (19) \\
\hline $\begin{array}{l}\text { Barrett's } \\
\quad \text { esophagus } \\
(n=20)\end{array}$ & $\begin{array}{l}\text { Oral consumption as } \\
\text { Lyophilized black } \\
\text { raspberries in water } \\
\text { suspension }\end{array}$ & $\begin{array}{l}32 \text { to } 45 \mathrm{~g} \mathrm{BRB} \\
1 \times \text { daily }\end{array}$ & 6 months & $\begin{array}{l}\text { Cell proliferation } \\
\text { Oxidative damage } \\
\text { Lipid peroxidation } \\
\text { Cholesterol } \\
\text { GST-pi, CDX2, } \\
\quad \text { NF-кB } \\
\text { Ellagitannin } \\
\quad \text { metabolites }\end{array}$ & $(20,21)$ \\
\hline $\begin{array}{l}\text { Colon Cancer } \\
(n=20)\end{array}$ & $\begin{array}{l}\text { Oral consumption as } \\
\text { Lyophilized black } \\
\text { raspberries in water } \\
\text { suspension }\end{array}$ & $\begin{array}{l}20 \mathrm{~g} \mathrm{BRB} \\
3 \times \text { daily (oral) }\end{array}$ & $1-9$ weeks & $\begin{array}{l}\text { DNA methylation } \\
\text { Cell proliferation } \\
\text { Apoptosis } \\
\text { Angiogenesis } \\
\beta \text {-Catenin,E- } \\
\text { Cadherin, c-Myc } \\
\text { Cyclin D1 } \\
\text { Metabolites }\end{array}$ & $(22,23,24)$ \\
\hline $\begin{array}{l}\text { Familial } \\
\text { adenomatous } \\
\text { polyposis } \\
(n=14)\end{array}$ & $\begin{array}{l}\text { Local delivery as } \\
\text { rectal suppository } \\
\text { alone }(n=7) \text { or in } \\
\text { combination w/ oral } \\
\text { consumption of } \\
\text { Lyophilized black } \\
\text { raspberries in water } \\
\text { suspension }(n=7)\end{array}$ & $\begin{array}{l}1.4 \mathrm{~g} / \mathrm{d} \text { BRB } \\
\text { suppository } \\
\text { alone or in } \\
\text { combination w/ } \\
20 \mathrm{~g} \mathrm{BRB} \\
3 \times \text { daily (oral) }\end{array}$ & 9 months & $\begin{array}{l}\text { Polyp number, burden } \\
\quad \& \text { size } \\
\text { DNA methylation } \\
\text { APC SNPs } \\
\text { Cell proliferation }\end{array}$ & (25) \\
\hline
\end{tabular}

$\mathrm{BRB}$, abbreviation for black raspberry.

and is estimated to impact over 60 million Americans. In addition, obesity imparts a 1.5 to 2.0 -fold increase risk for $\mathrm{BE}$ and a 2 to 2.5 -fold increase risk for EAC [36]. Thus, there is a large population at risk for BE and potential progression to EAC. Plant based diets rich in fruits, vegetables, and fiber are associated with reduced risk for EAC [1]. A 6 month pilot study was conducted to assess the long-term tolerability of a food based chemopreventive approach and to investigate whether BRB modulate oxidative damage and other aberrant 
signaling cascades associated with GERD and progression of Barrett's esophagus. Details of the study approach and eligibility have been previously reported [20,21]. In brief, all subjects were adults, 18 years of age or older with a diagnosis of Barrett's esophagus $(\geq 1 \mathrm{~cm})$ on the current and two previous endoscopies. Twenty patients were enrolled with each essentially serving as their own control. Lyophilized or freeze dried BRB powder was administered at 32 and $45 \mathrm{~g} 1 \times$ daily to women and men, respectively. This gram quantity is approximately equivalent to 1.5 and 2 cups of whole fruit and was based on early preclinical research in animal models showing that 5 and $10 \%$ BRB in the diet inhibited esophageal cancer [5-9]. Patients mixed the BRB powder with about 6 ounces of water and consumed the mixture orally each morning. Urinary markers were assessed at baseline or pre-treatment and at 12 and 26 weeks post-BRB administration. Specific measurements included urinary excretion of 8-epi-prostaglandin F2 $\alpha$ (8-PGF2 $\alpha$ ) and 8-hydroxy-2'-deoxyguanosine (8-OHdG), markers of lipid peroxidation and oxidative DNA damage. Urinary levels of the ellagitannin metabolites, Urolithin A-glucuronide, Urolithin A-sulfate and dimethylellagic acid glucuronide (DMEAG) were also investigated for the first time, as potential markers of compliance. Esophageal and gastric tissues were assessed for changes in markers linked to proliferation, differentiation, detoxification and inflammation. Specific immunohistochemical markers assessed in esophageal tissues included Ki-67, CDX2, GST-pi, and NF-кB. In addition, secondary outcomes included changes in histopathology, Barrett's esophageal length, blood pressure, total cholesterol levels and body mass index $[20,21]$.

Findings from this study showed that BRB were well tolerated at 32 and $45 \mathrm{~g} /$ daily for 6 months and that compliance was high with $96 \%$ consuming BRB daily, based on intake records and counts from returned packages. Levels of the ellagitannin metabolite, Urolithin A-glucuronide, significantly increased following BRB treatment for 12 and 26 weeks compared to baseline [20,21]. At baseline 15\% of patients expressed detectable levels of Urolithin A-glucuronide [70 ng/mL]; whereas, 85\% of patients expressed elevated levels at week 12 [1287 ng/mL] and week 26 [1723 ng/mL] of study supporting that urolithin A-glucuronide may serve as an additional indicator of compliance. BRB consumption resulted in elevated levels of urinary urolithin A-sulfate and DMEAG; however, these metabolites were not impacted to the same degree or magnitude [21]. Differences in metabolizing enzymes may be responsible for variations in response among patients, but additional research is warranted to better understand the role of individual differences in metabolizing enzymes and potential interaction with the microbiome profile of individual patients.

BRB consumption reduced urinary excretion of 8 -PGF $2 \alpha$ following 12 weeks, reaching statistical significance by 26 weeks of treatment. This marker is considered a reliable and relatively stable marker of overall oxidative stress levels and more specifically an indicator of lipid peroxidation. Reductions in 8-PGF2 $\alpha$ are especially promising, considering that average BMIs increased significantly over the 6 month trial. BRB consumption did not significantly reduce levels of $8-\mathrm{OHdG}$, a marker of DNA damage and there was greater variability noted for this marker with increases noted among 10 patients and decreases among 6 patients over the study duration [20, 21]. Interestingly, total cholesterol levels non-significantly declined in BRB treated patients, particularly those who presented with baseline cholesterol levels $>200 \mathrm{mg} / \mathrm{dL}(p=0.165)$. Cholesterol levels reduced from $234 \mathrm{mg} / \mathrm{dL}$ at baseline to $226 \mathrm{mg} / \mathrm{dL}$ at week 26, following BRB treatment [21]. The latter finding is in alignment with other recent studies pointing to the positive effects of berry consumption on indicators of cardiovascular health, even in patients with metabolic syndrome [37]. Jeong and colleagues recently reported that a BRB extract administered to patients with metabolic syndrome significantly reduced total cholesterol and cytokines including IL-6 and TNF- $\alpha$ levels following 12 weeks of consumption [37]. Obesity is a strong risk factor for both BE and EAC [35, 36]; thus, agents which positively impact risk factors, as well as defined cancer processes hold particular promise. In terms of tissue specific changes, the main positive effect was increased expression of the detoxification marker GST-pi in BE epithelium of 55.6\% of patients. Overall proliferation rates as measured by $\mathrm{Ki}-67$ were unchanged with decreased levels noted in two patients and increased levels in 2 other patients [21]. There were no significant changes in BE histopathology grade or length of the BE tongue following treatment. However, it is important to note that detection of a $30 \%$ or $1 \mathrm{~cm}$ change in the length of the BE segment would require a patient sample size of 43 ; thus, this pilot study was under powered for detecting changes in BE length. 
Taken together, these results support that BRB consumed at 32 to $45 \mathrm{~g} /$ day positively impact plasma metabolites including total cholesterol and increased urinary ellagitannin metabolites, reduced a urinary marker of lipid peroxidation, and importantly at the tissue level significantly increased GST-pi levels, a marker of detoxification [21]. Potentially higher doses, more frequent administration or an alternative delivery matrix is required for modulation of additional tissue specific markers.

\subsection{BRB in colorectal cancer patients}

Colorectal cancer (CRC) is the second leading cause of cancer related deaths with over 49,000 cases expected in the US this year [34]. Over the last 20 years improvements in screening and treatment modalities have led to decreases in the colorectal cancer death rates. Despite these advances approximately half of patients diagnosed with CRC will eventually die of the disease $[1,2]$. Thus, there remains a critical need for more effective preventive and treatment options targeting colorectal cancer. It is estimated that $50 \%$ of colorectal cancers could be prevented through exercising and maintaining a healthy weight, limiting consumption of red and processed meat and eating a plant based diet rich in vegetables, whole grains and fruit $[1,4]$. Positive preclinical results support that dietary BRB significantly inhibited the formation of chemically induced colon tumors $[10,11]$. A clinical trial was initiated enrolling 20 colorectal cancer patients. Exclusion and inclusion criteria were previously published [22-24]. All patients had tissues taken before and after 1 to 9 weeks of oral BRB ( $20 \mathrm{~g} 3 \times$ daily) consumption. Changes in markers associated with cell proliferation, apoptosis, angiogenesis and Wnt signaling were measured. Positive findings were noted in patients who consumed BRB for 4 or more weeks. Specific findings included BRB altered methylation of DNMT1, SFRP2, and PAX6a in normal adjacent tissues and DNMT1, SFRP2, PAX6a and WIF1 in colorectal tissues [22-24]. BRB did not induce changes in global methylation. Immunohistochemical staining results showed that BRB modulated $\beta$-catenin, Ki-67, TUNEL, CD105, and DNMT1 in colorectal tissue and CD105 and DNMT1 in normal tissues [22-24]. The fact that BRB significantly altered a number of markers in key molecular pathways known to be altered in colon cancer is encouraging and it is notable that the effect occurred in a relatively short period of time. Positive effects were found for many of the outcomes in patients who had been consuming BRB for 4 or more weeks supporting that even short-term or potentially intermittent use of agents with cancer inhibitory potential may offer benefits.

Research findings from the above colorectal trial were extended to include BRB induced modulation of select plasma cytokines [23] as well as metabolic profiles in the plasma and urine following treatment [24]. Significant changes in plasma levels of GM-CSF and IL-8 were reported in patients consuming berries for more than 10 days and changes correlated with apoptosis induction and reduced proliferation in colorectal tissues [23]. These results support that BRB induce changes in circulatory markers rather rapidly (10 days), but that tissue specific alterations require a longer duration of BRB administration ( 4 or more weeks) or higher cumulative dose. These results are consistent with those from the BE study [21]. A non-targeted metabolic analysis uncovered over 400 annotated metabolites, with 34 and 16 metabolites significantly changed by BRB in the urine and plasma, respectively [24]. Specific metabolic pathways altered with BRB treatment include amino acid metabolism, lipid metabolism and xenobiotics. BRB polyphenols were metabolized to multiple benzoate species which was associated with an enhanced amino acid metabolite. Further, increased levels of 4-methycatechol sulfate in both urine and plasma correlated to increased apoptosis at the tissue level [24].

\subsection{BRB in patients with familial adenomatous polyposis}

Familial adenomatous polyposis (FAP) is an inherited disorder characterized by early onset of colonic polyposis and progression to cancer of the colon and rectum by age $40[39,40]$. FAP is due to germline mutations in the $A P C$ gene and requires patients to be closely monitored via endoscopic surveillance for early detection of polyps. Once polyps manifest the only effective management to prevent cancer is eventual colectomy; thus, 
agents that delay polyp progression may offer alternative or complementary preventive options. Non-steroidal anti-inflammatory drugs (NSAIDS) regress FAP associated polyps, but due to associated gastrointestinal, cardiovascular and cerebrovascular events non-toxic alternative strategies are being investigated. Thus, a small pilot study enrolled 14 patients with FAP to assess the potential beneficial effects of BRB on measurements of polyp number, size, and burden. BRB were administered daily for a period of 9 months. Arm 1 consisted of 7 patients consuming a maltodextrin placebo powder three times a day combined with two BRB rectal suppositories $(0.7 \mathrm{~g}$ each administered at bedtime). Arm 2 included the remaining 7 patients who consumed freeze dried BRB powder ( $20 \mathrm{~g} 3 \times$ daily) mixed with water, plus two BRB rectal suppositories $(0.7 \mathrm{~g}$ each administered at bedtime). Study specifics have been previously reported [25]. In brief, inclusion criteria included: $\geq 18$ years of age, a diagnosis of FAP with at least 5 rectal polyps $>2 \mathrm{~mm}$ at baseline endoscopy and no NSAID use currently or in the last 2 months. Prior to treatment initiation and at 36 weeks of study each patient underwent flexible sigmoidoscopy to record polyp number, size, and burden defined as the sum of diameters of all adenomas $>2 \mathrm{~mm}$. Up to two polyps were removed at baseline for biomarker analysis and all polyps were harvested at 36 weeks of study. Additional outcomes includedimmunohistochemical analysis of Ki-67 as a marker of cellular proliferation, TUNEL staining for apoptosis, cMyc, DNMT1, p16 and analysis of DNA methylation [25].

Combining the two treatment arms, study results showed a significant reduction in tumor burden post-treatment with BRB suppositories [25]; however, the authors concluded that there was no additional benefit for patients consuming BRB orally. Interestingly, the magnitude of change from baseline polyp burden does appear greater given the combined treatment (about 75\%) compared to suppositories alone treatment (about 40\%), raising the question of whether there is a benefit in terms of magnitude of effect. It is likely that a sample size greater than 7 is needed to assess whether combined oral consumption of BRB with suppository treatment offers any increased efficacy compared to suppositories alone. Certainly, as discussed in the context of trials targeting oral premalignancy, the use of suppositories may offer the advantage of local delivery depending on polyp location; however, expanded studies are necessary to determine whether combined oral and direct delivery approaches offer any advantages for FAP patients. Three patients presented with increases in polyp number and burden following treatment completion; one patient in the suppository alone arm and two patients on the combined treatment arm [25]. Patients that responded to BRB treatment as evidenced by decreased tumor burden also showed reductions in cell proliferation rates as measured by Ki-67, decreased DNMT1, increased P16 levels, yet, cMYC and TUNEL expression were reportedly unchanged by BRB treatment [25]. BRB treatment resulted in decreased $p 16$ promoter methylation, but did not differentially impact Wnt pathway antagonists, SFRP2 and WIF1 in polyps from responders versus non-responders. Responders were also investigated for differences in somatic SNPs in the APC gene, but no differences were detected [25].

These results are encouraging and in alignment with earlier research conducted in colon cancer patients in terms of p16 findings, but unique in that Wnt regulators were not differentially altered between responders and non-responders [25]. Still, this study did detect that miRNAs regulating Wnt pathways were demethylated by $\mathrm{BRB}$ in adenomas from responders. Collectively, the data utilizing BRB to target colon cancers is highly positive showing inhibitory effects in both inherited FAP associated and non-FAP associated colon cancers. Once again, the results support that the cohort of FAP patients appeared to have patients that responded to BRB exposure and a subpopulation of non-responders.

\section{Discussion/Conclusion}

Evidence between single food sources and cancer protection is limited and challenging to study. To date, the strongest cancer inhibitory evidence exists for food groups and especially plant based diets rich in fruits, vegetables and fiber [1]. In terms of extracts, much of the evidence for cancer protection is strongest the closer the extract mimics the whole plant components [42-44]. To determine the esophageal cancer inhibitory contribution made by BRB versus BRB derived fractions, a preclinical in vivo study was conducted in F344 rats comparing a 5\% 
lyophilized black raspberry powder to a BRB derived anthocyanin rich fraction, a BRB derived ethanol/water soluble fraction, a BRB derived insoluble fraction, a BRB derived hexane extract and a BRB derived sugar fraction [42]. The results supported that the 5\% BRB, the anthocyanin fraction and ethanol extract which all contained similar levels of anthocyanins were equally effective in inhibiting NMBA-induced esophageal cancer [42] supporting the historical rationale that anthocyanins are the cancer inhibitory component of BRB. However, the BRB derived insoluble or residue fraction was nearly as inhibitor and this fraction contains very low levels of anthocyanins [42] illustrating the complex nature of individual food products and the fact that many foods of interest are not fully characterized from a cancer inhibitory standpoint.

A clinical investigation evaluating red and yellow tomatoes versus similar levels of lycopene in the form of a purified extract reported short term tomato intake induces serum changes that favorably modulate cancer-related gene expression profiles in treated cell lines, including the yellow or lycopene-free tomato; whereas, purified lycopene had both positive and potentially deleterious effects as evidenced by up-regulation of pro-cancer genes [43]. Thus, identification of the main or sole component from a fruit or food matrix responsible for cancer inhibition remains challenging despite technological advancements.

Still, the combination of preclinical and early clinical studies utilizing various black raspberry products have revealed beneficial effects linked to cancer inhibition in select targets as summarized herein [17-25]. Completed published studies evaluating BRB in the clinical setting report positive effects on preneoplastic lesions or cancers of the head and neck, esophagus and colon. The positive effects of BRB in clinical evaluations include: antiproliferative effects; activation of pro-cell death pathways; histologic regression of oral intraepithelial neoplasia associated with improved histologic grade and significantly reduced $\mathrm{LOH}$ at tumor suppressor gene associated loci, reduced suppression of genes linked to RNA processing, growth factor recycling, and reduced COX-2 levels in the oral cavity; in the colon, inhibition of FAP-associated polyp progression, demethylation of known tumor suppressor genes (Wnt pathway antagonists and p16) and improved plasma cytokine profiles (GM-CSF, IL-8) were reported; in Barrett's patients, increased tissue levels of GST-pi, decreased lipid peroxidation/oxidative stress (urinary 8-isoprostane levels) and reduced cholesterol levels followed BRB treatment. In addition, other studies of obese patients have reported improved serum cytokine levels and lipid profiles including IL- 6 , TNF- $\alpha$, and cholesterol [34]. Results have not all been uniform across studies or even within, most studies report a percentage of responders and non-responders within the cohort evaluated, likely reflecting the heterogeneity between subjects and the complexity of each cancer or precursor lesion. In addition, the mode, delivery matrix, concentration, frequency and duration of black raspberry delivery has varied across studies, potentially contributing to divergent outcomes. BRB have been administered as a freeze dried powder suspended in water prior to consumption (37 to $60 \mathrm{~g} /$ day $)$, as a rectal suppository alone $(1.4 \mathrm{~g} /$ day $)$ or in combination with oral delivery $(60 \mathrm{~g} /$ day $)$ and as a topical agent $(10 \% \mathrm{w} / \mathrm{w})$ delivered in a bioadhesive gel in the oral cavity. Studies conducted in patients with FAP or oral premalignancy permit relative direct delivery of BRB bioactive constituents to the target area. This has the advantage of requiring less product, increasing direct contact time and takes advantage of local metabolism compared with oral delivery of the suspended freeze-dried powder which allows less direct contact and relatively more systemic benefits. Still, common themes across studies have emerged including that BRB have anti-inflammatory effects, reduce oxidative stress, impact metabolism and restore tumor suppressive activity in a target specific manner resulting in regression of oral cavity and colon lesions.

The precise dose and duration of BRB required for optimum cancer inhibitory effects remains to be elucidated, but results to date suggest lower concentrations may favorably impact lipid profiles, while potentially higher concentrations are required for tissue specific effects. In addition, the lengths of studies have ranged from 1 week to 9 months with data supporting that longer duration studies of at least 4 weeks to 3 months or beyond have greater impact. Further research is warranted to increase our knowledge of BRB cancer inhibitory mechanisms and to improve our ability to discern which patient populations are likely to benefit from BRB administration. A marker of compliance to reliably link intake to effects would be valuable in future clinical trials as well. Ellagitannin metabolites were included in the BE trial, but were expressed in only $85 \%$ of the BE patients illustrating the complexity and limitations of the approach of utilizing metabolites which may vary among 
patients due to inherent genetic or metabolic variation. An alternative approach that should be considered in future prospective trials is to add a food grade standard to BRB products permitting more accurate compliance measurements.

Progress has been made with BRB as well as other polyphenols in terms of understanding bioavailability and metabolism, but additional work is needed. Kay and colleagues utilized 13C- labeling approach to evaluate absorption, metabolism and excretion of cyanidin-3-glucoside in blood, urine and fecal samples following administration of a single $500 \mathrm{mg}$ dose. This study uncovered a number of new metabolites offering improved understanding of compound fate. Additional studies incorporating these approaches with additional polyphenol metabolites, in varied matrices and following longer periods of administration would be highly beneficial. In addition, there is interest and active research focused on developing novel products for delivering food-based agents targeting cancer prevention which may offer alternative modes or matrices of delivery, especially to highly accessible targets. As an example, pectin and starch based black raspberry confections retain $93 \%$ of original anthocyanins compared to only $60 \%$ in a hard candy confection designed to delivery phytochemicals [45].

In conclusion, human clinical trials utilizing BRB have demonstrated chemopreventive effects in the oral cavity, esophagus and colon. However, additional research is warranted and should include 1) assessments of other more readily available berries that have demonstrated beneficial health effects, alone or in combination with BRB; 2) comparison of the whole BRB fruit versus dominant constituents and active metabolites; 3) optimizing BRB delivery mode and matrices; 4) factors impacting BRB metabolism and bioavailability [46, 47]; 5) effects BRB on cancer-initiating cells or cancer stem cells [48]; 6) BRB treatment as it relates to phase of carcinogenesis; and 7) whether BRB improve chemotherapeutic or radiation efficacy.

\section{Acknowledgments}

Thanks to all the patients who participated in the clinical trials summarized.

\section{References}

[1] World Cancer Research Fund / American Institute for Cancer Research. Food, Nutrition, Physical Activity, and the Prevention of Cancer: A Global Perspective [Internet]. Washington DC: AICR; 2007. Available from: http://www.aicr.org/assets/docs/pdf/reports/Second_Expert_Report.pdf.

[2] Turati F, Rossi M, Pelucchi C, Levi F, La Vecchia C. Fruit and vegetables and cancer risk: A review of southern European studies. Br J Nutr. 2015;113(Suppl 2):S102-10.

[3] Bravi F, Edefonti V, Randi G, Ferraroni M, La Vecchia C, Decarli A. Dietary patterns and upper aerodigestive tract cancers: An overview and review. Ann Oncol. 2012;23(12):3024-39.

[4] Norat T, Aune D, Chan D, Romaguera D. Fruits and vegetables: Updating the epidemiologic evidence for the WCRF/AICR lifestyle recommendations for cancer prevention. Cancer Treat Res. 2014;159:35-50.

[5] Kresty LA, Morse MA, Morgan C, Carlton PS, Lu J, Gupta A, et al. Chemoprevention of esophageal tumorigenesis by dietary administration of lyophilized black raspberries. Cancer Res. 2001;61(16):6112-9.

[6] Lechner JF, Reen RK, Dombkowski AA, Cukovic D, Salagrama S, Wang LS, et al. Effects of a black raspberry diet on gene expression in the rat esophagus. Nutr Cancer. 2008;60(Suppl 1):61-9.

[7] Stoner GD, Chen T, Kresty LA, Aziz RM, Reinemann T, Nines R. Protection against esophageal cancer in rodents with lyophilized berries: Potential mechanisms. Nutr Cancer. 2006;54(1):33-46.

[8] Liston BW, Gupta A, Nines R, Carlton PS, Kresty LA, Harris GK, et al. Incidence and effects of Ha-ras codon 12 G->A transition mutations in preneoplastic lesions induced by N-nitrosomethylbenzylamine in the rat esophagus. Mol Carcinog. 2001;32(1):1-8.

[9] Peiffer DS, Zimmerman NP, Wang LS, Ransom BW, Carmella SG, Kuo CT, et al. Chemoprevention of esophageal cancer with black raspberries, their component anthocyanins, and a major anthocyanin metabolite, protocatechuic acid. Cancer Prev Res (Phila). 2014;7(6):574-84. 
[10] Harris GK, Gupta A, Nines RG, Kresty LA, Habib SG, Frankel WL, et al. Effects of lyophilized black raspberries on azoxymethaneinduced colon cancer and 8-hydroxy-2'- deoxyguanosine levels in the Fischer 344 rat. Nutr Cancer. 2001;40(2):125-33.

[11] Wang LS, Kuo CT, Huang TH, Yearsley M, Oshima K, Stoner GD, et al. Black raspberries protectively regulate methylation of Wnt pathway genes in precancerous colon tissue. Cancer Prev Res (Phila). 2013;6(12):1317-27.

[12] Casto BC, Kresty LA, Kraly CL, Pearl DK, Knobloch TJ, Schut HA, et al. Chemoprevention of oral cancer by black raspberries. Anticancer Res. 2002;22(6C):4005-15.

[13] Aiyer HS, Gupta RC. Berries and ellagic acid prevent estrogen-induced mammary tumorigenesis by modulating enzymes of estrogen metabolism. Cancer Prev Res (Phila). 2010;3(6):727-37.

[14] Duncan FJ, Martin JR, Wulff BC, Stoner GD, Tober KL, Oberyszyn TM, et al. Topical treatment with black raspberry extract reduces cutaneous UVB-induced carcinogenesis and inflammation. Cancer Prev Res (Phila). 2009;2(7):665-72.

[15] Stoner GD. Foodstuffs for preventing cancer: The preclinical and clinical development of berries. Cancer Prev Res (Phila). 2009;2(3):187-94.

[16] Czank C, Cassidy A, Zhang Q, Morrison DJ, Preston T, Kroon PA, et al. Human metabolism and elimination of the anthocyanin, cyanidin-3-glucoside: A (13)C-tracer study. Am J Clin Nutr. 2013;97(5):995-1003.

[17] Shumway BS, Kresty LA, Larsen PE, Zwick JC, Lu B, Fields HW, et al. Effects of a topically applied bioadhesive berry gel on loss of heterozygosity indices in premalignant oral lesions. Clin Cancer Res. 2008;14(8):2421-30.

[18] Mallery SR, Zwick JC, Pei P, Tong M, Larsen PE, Shumway BS, et al. Topical application of a bioadhesive black raspberry gel modulates gene expression and reduces cyclooxygenase 2 protein in human premalignant oral lesions. Cancer Res. 2008;68(12):4945-57.

[19] Mallery SR, Tong M, Shumway BS, Curran AE, Larsen PE, Ness GM, et al. Topical application of a mucoadhesive freeze-dried black raspberry gel induces clinical and histologic regression and reduces loss of heterozygosity events in premalignant oral intraepithelial lesions: Results from a multicentered, placebo-controlled clinical trial. Clin Cancer Res. 2014;20(7):1910-24.

[20] Kresty LA, Frankel WL, Hammond CD, Baird ME, Mele JM, Stoner GD, et al. Transitioning from preclinical to clinical chemopreventive assessments of lyophilized black raspberries: Interim results show berries modulate markers of oxidative stress in Barrett's esophagus patients. Nutr Cancer. 2006;54(1):148-56.

[21] Kresty LA, Fromkes JJ, Frankel WL, Hammond CD, Seeram NP, Baird ME, Stoner GD. A phase I pilot study evaluating the beneficial effects of black raspberries in patients with Barrett's esophagus. Oncotarget 2016, In Press.

[22] Wang LS, Arnold M, Huang YW, Sardo C, Seguin C, Martin E, et al. Modulation of genetic and epigenetic biomarkers of colorectal cancer in humans by black raspberries: A phase I pilot study. Clin Cancer Res. 2011;17(3):598-610.

[23] Mentor-Marcel RA, Bobe G, Sardo C, Wang LS, Kuo CT, Stoner G, et al. Plasma cytokines as potential response indicators to dietary freeze-dried black raspberries in colorectal cancer patients. Nutr Cancer. 2012;64(6):820-5.

[24] Pan P, Skaer CW, Stirdivant SM, Young MR, Stoner GD, Lechner JF, et al. Beneficial regulation of metabolic profiles by black raspberries in human colorectal cancer patients. Cancer Prev Res (Phila). 2015;8(8):743-50.

[25] Wang LS, Burke CA, Hasson H, Kuo CT, Molmenti CL, Seguin C, et al. A phase Ib study of the effects of black raspberries on rectal polyps in patients with familial adenomatous polyposis. Cancer Prev Res (Phila). 2014;7(7):666-74.

[26] Lucenteforte E, Garavello W, Bosetti C, La Vecchia C. Dietary factors and oral and pharyngeal cancer risk. Oral Oncol. 2009;45(6): 461-7.

[27] Meurman JH. Infectious and dietary risk factors of oral cancer. Oral Oncol. 2010;46(6):411-413.

[28] Mallery SR, Stoner GD, Larsen PE, Fields HW, Rodrigo KA, Schwartz SJ, et al. Formulation and in-vitro and in-vivo evaluation of a mucoadhesive gel containing freez dried black raspberries: Implications for oral cancer chemoprevention. Pharm Res. 2007;24:728737.

[29] Ugalde CM, Liu Z, Ren C, Chan KK, Rodrigo KA, Ling Y, et al. Distribution of anthocyanins delivered from a bioadhesive black raspberry gel following topical intraoral application in normal healthy volunteers. Pharm Res. 2009;26(4):977-86.

[30] Kresty LA, Mallery SR, Knobloch TJ, Song H, Lloyd M, Casto BC, et al. Alterations of p16(INK4a) and p14(ARF) in patients with severe oral epithelial dysplasia. Cancer Res. 2002;62(18):5295-300.

[31] Neville BW, Day TA. Oral cancer and precancerous lesions. CA Cancer J Clin. 2002;52(4):195-215.

[32] Silverman S, Jr, Gorsky M, Lozada F. Oral leukoplakia and malignant transformation. A follow-up study of 257 patients. Cancer. 1984;53(3):563-8.

[33] Manach C, Williamson G, Morand C, Scalbert A, Rémésy C. Bioavailability and bioefficacy of polyphenols in humans. I. Review of 97 bioavailability studies. Am J Clin Nutr. 2005;81:230S-42S.

[34] American Cancer Society. Cancer Facts \& Figures 2016 [Internet]. Atlanta: American Cancer Society; 2016. Available from: http://www.cancer.org/acs/groups/content/@editorial/documents/document/acspc- 044552.pdf

[35] Holmes RS, Vaughan TL. Epidemiology and pathogenesis of esophageal cancer. Semin Radiat Oncol. 2007;17(1):2-9. 
[36] Hampel H, Abraham NS, El-Serag HB. Meta-analysis: Obesity and the risk for gastroesophageal reflux disease and its complications. Ann Intern Med. 2005;143(3):199-211.

[37] Jeong HS, Hong SJ, Lee TB, Kwon JW, Jeong JT, Joo HJ, et al. Effects of black raspberry on lipid profiles and vascular endothelial function in patients with metabolic syndrome. Phytother Res. 2014;28(10):1492-8.

[38] Gu J, Ahn-Jarvis JH, Riedl KM, Schwartz SJ, Clinton SK, Vodovotz Y. Characterization of black raspberry functional food products for cancer prevention human clinical trials. J Agric Food Chem. 2014;62(18):3997-4006.

[39] National Cancer Institute. SEER Cancer Statistics Factsheets: Colon and Rectum Cancer [Internet]. Bethesda: National Cancer Institute; 2015. Available from: http://seer.cancer.gov/statfacts/html/colorect.html.

[40] Ellebaek E, Andersen MH, Svane IM, Straten PT. Immunotherapy for metastatic colorectal cancer: Present status and new options. Scand J Gastroenterol. 2012;47(3)315-24.

[41] Standard Task F, American Society of C, Rectal S, Collaborative Group of the Americas on Inherited Colorectal C. Practice parameters for the identification and testing of patients at risk for dominantly inherited colorectal cancer. Dis Colon Rectum. 2001;44(10):1403.

[42] Wang LS, Hecht SS, Carmella SG, Nanxiong Y, Larue B, Henry C, McIntyre C. et al. Anthocyanins in black raspberries prevent esophageal tumors in rats. Cancer Prev Res. 2009;2(1):84-93.

[43] Talvas J, Caris-Veyrat C, Guy L, Rambeau M, Lyan B, Minet-Quinard R, Lobaccaro JM. et al. Differential effects of lycopene consumed in tomato paste and lycopene in the form of a purified extract on target genes of cancer prostatic cells. Am J Clin Nutr. 91(6): 1716-24.

[44] Seeram NP, Adams LS, Henning SM, Niu Y, Zhang Y, Nair MG. et al. In vitro antiproliferative, apoptotic and antioxidant activities of punicalagin, ellagic acid and a total pomegranate tannin extract are enhanced in combination with other polyphenols as found in pomegranate juice. J Nutr Biochem. 2005;16(6):360-7.

[45] Gu J, Ahn-Jarvis JH, Vodovotz Y. Development and characterization of different black raspberry confection matrices designed for delivery of phytochemicals. J Food Sci. 2015;80(3):E610-8.

[46] Cerella C, Radogna F, Dicato M, Diederich M. Natural compounds as regulators of the cancer cell metabolism. Int J Cell Biol. 2013;2013:639401.

[47] Bohn T. Dietary factors affecting polyphenol bioavailability. Nutr Rev. 2014;72(7):429-52.

[48] Pistollato F, Giampieri F, Battino M. The use of plant-derived bioactive compounds to target cancer stem cells and modulate tumor microenvironment. Food Chem Toxicol. 2015;75:58-70. 